\title{
Suspended Decoupler: A New Design of Hydraulic Engine Mount
}

\author{
J. Christopherson, ${ }^{1}$ M. Mahinfalah, ${ }^{2}$ and Reza N. Jazar ${ }^{3}$ \\ ${ }^{1}$ MTS Systems Corporation, 14000 Technology Drive, Eden Prairie, MN 55344-2290, USA \\ ${ }^{2}$ Department of Mechanical Engineering, Milwaukee School of Engineering, Milwaukee, WI 53202, USA \\ ${ }^{3}$ School of Aerospace, Mechanical, and Manufacturing Engineering, RMIT University, Melbourne, VIC 8083, Australia \\ Correspondence should be addressed to M. Mahinfalah, mahinfalah@msoe.edu
}

Received 28 June 2011; Accepted 14 September 2011

Academic Editor: Mohammad Tawfik

Copyright ( $\odot 2012$ J. Christopherson et al. This is an open access article distributed under the Creative Commons Attribution License, which permits unrestricted use, distribution, and reproduction in any medium, provided the original work is properly cited.

Because of the density mismatch between the decoupler and surrounding fluid, the decoupler of all hydraulic engine mounts (HEM) might float, sink, or stick to the cage bounds, assuming static conditions. The problem appears in the transient response of a bottomed-up floating decoupler hydraulic engine mount. To overcome the bottomed-up problem, a suspended decoupler design for improved decoupler control is introduced. The new design does not noticeably affect the mechanism's steady-state behavior, but improves start-up and transient response. Additionally, the decoupler mechanism is incorporated into a smaller, lighter, yet more tunable and hence more effective hydraulic mount design. The steady-state response of a dimensionless model of the mount is examined utilizing the averaging perturbation method applied to a set of second-order nonlinear ordinary differential equations. It is shown that the frequency responses of the floating and suspended decoupled designs are similar and functional. To have a more realistic modeling, utilizing nonlinear finite elements in conjunction with a lumped parameter modeling approach, we evaluate the nonlinear resorting characteristics of the components and implement them in the equations of motion.

\section{Introduction and Statement of Problem}

Modern vehicles illustrate a trend toward lighter, higher performance, aluminum-based engines thereby increasing the potential for vibration. The engine is the largest concentrated mass in a vehicle and causes vibration if it is not properly isolated and constrained. The trend for many years to isolate vibrations was to simply connect the engine and frame by means of an engine mount made of elastomeric materials such as rubber [1-3]. Modeling the rubber isolator by a linear system and considering a base excited single-degree-offreedom system, we know in the frequency response curves of the acceleration transmitted to the isolated mass there exists a crossing point at a frequency ratio value of $\omega / \omega_{n}=2^{1 / 2}$ in which all the curves representing systems with differing damping ratios converge [4]. This is a switching point for systems where system behavior reverses dependent upon excitation frequency. This paradoxical behavior indicates that for optimum isolation of a structure from acceleration, hence force, a mount is needed in which high damping is allowed at low excitation frequencies, and low damping is allowed at increased excitation frequencies.

Because there is a need for a vibration isolator that can exhibit a dual damping ratio that is dependent upon frequency the hydraulic engine mount was introduced. The hydraulic engine mount is a device that approximately provides the desired damping characteristics via the implementation of a mechanical switching mechanism known as the decoupler in conjunction with a narrow, highly restrictive fluid path known as the inertia track $[1,2$, 5-9]. These two mechanisms act together, assuming an appropriately designed system, to provide a passive means of variable damping dependent upon excitation characteristics [10]. More specifically, when a large pressure differential is imparted to the fluid chambers, by means of a substantial outside perturbation, the decoupler will bottom out in its cage bounds and cause the pressure differential within the mount to be equalized via the inertia track. Due to the inertia tracks dimensions it provides an increased damping coefficient to the engine mount. However, when the external 


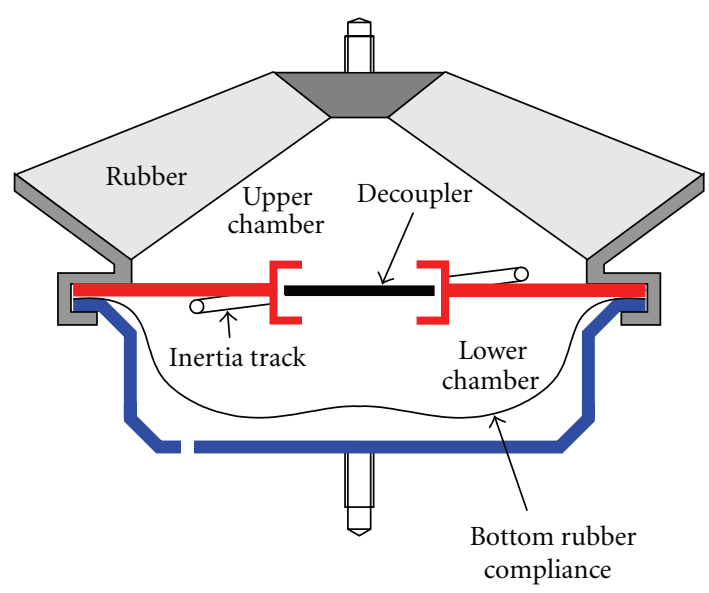

(a)

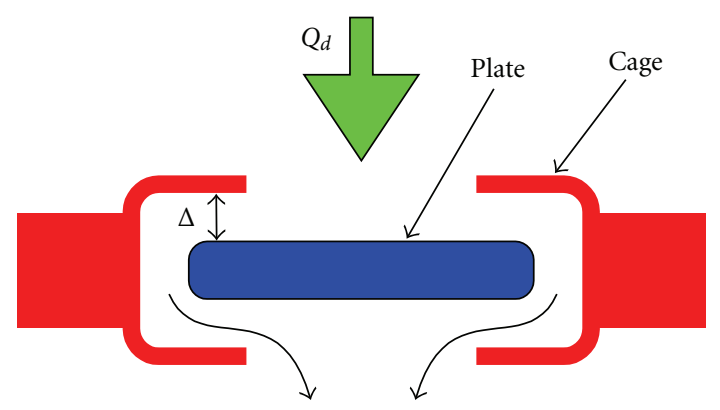

(b)

Figure 1: (a) Typical hydraulic engine mount. (b) Typical decoupler mechanism.

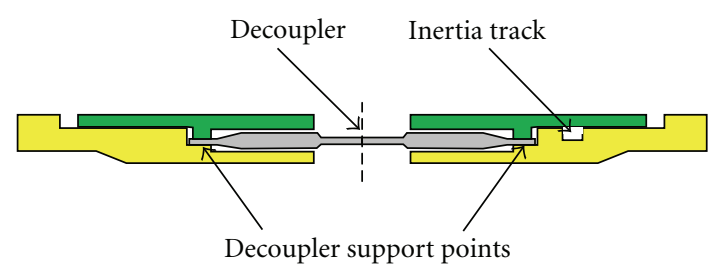

Figure 2: New decoupler mechanism.

perturbation is low in intensity, or at increased frequency, the decoupler does not bottom out, and hence the inertia track is effectively short-circuited; therefore, due to the decoupler's large dimensions, the system provides a low damping coefficient.

Figure 1(a) illustrates a schematic of a typical floatingdecoupler hydraulic engine mount (HEM) $[9,10]$. The engine mount is named as such because the decoupler "floats" freely inside of its housing. The basic premise of operation of the HEM is relatively straightforward. The engine is supported by a rubber structure acting as the main load carrying component, and a means by which to induce fluid motion within the engine mount [2]. The fluid motion induced in the engine mount, due to external excitation, is then forced through a system of passageways of inertia track and decoupler. The preferred pathway is dependent upon the nature of the excitation.
The decoupler and its housing are shown in Figure 1(b). Large amplitude, low frequency excitations impart a significant enough fluid motion that the decoupler plate is forced to bottom out on its surrounding cage thereby forcing the fluid to flow through the inertia track into the compliant lower chamber. The inertia track is a long, small-diameter tube that runs circumferentially around the engine mount providing a very restrictive flow path between upper and lower chambers. Due to the restrictive nature of the inertia track, an increased viscous damping coefficient is realized for the system. This increased damping acts to reduce the acceleration transmissibility of the mount at low excitation frequencies. However, at increased excitation frequencies, the decoupler plate does not bottom out on the cage bounds. Instead it moves back and forth freely providing a relatively low flow restriction. Because the decoupler provides a low restriction to fluid flow, it becomes the preferred flow path, and acts on reducing the damping coefficient of the engine mount.

This system works quite well and is in place on the large majority of automotive applications to date. It is analyzed, and modeled by researchers since 1980 from different viewpoints. Adiguna and coworkers determined dynamic behavior of HEMs in time domain [11] and frequency domain [5], utilizing linear and nonlinear lumped models [12]. The nonlinear function of the decoupler is successfully modeled, examined, and applied by Golnaraghi and Jazar $[13,14]$ utilizing a third-degree equation to describe a nonlinear damping. Adapting their model, Christopherson and Jazar $[15,16]$ optimized a sprung mass suspended by an HEM and provided a design method.

In the present investigation we study two common assumptions and explore their effects in modeling and dynamics of hydraulic engine mounts. First assumption is that in lumped model of the system, the nonlinearities involved in elastomechanical parts are usually ignored and a linear behavior is assumed. Second assumption is that either in transient or steady-state responses it is assumed that the decoupler is settled down in its neutral position exactly in the middle of the gap of decoupler duct. Therefore, two questions arise as to what are the effects of nonlinearities involved in elastomechanical parts, and what happens on initial start up if the decoupler is bottomed up.

This investigation will utilize finite element analysis to determine the mechanical behavior of the components, and will employ perturbation analysis to determine transient and steady-state behaviors of the mount.

Because of the density mismatch between the decoupler and surrounding fluid, the decoupler will float, sink, twist, or stick to the cage bounds, assuming static conditions. The problem is what happens if the decoupler is in a nonoptimum location for a given random or initial excitation to provide either low damping by being open or closed to allow for high damping.

We introduce a supported decoupler mechanism illustrated in Figure 2. By supporting the decoupler it is ensured to be in a neutral location upon startup. However, the trick to designing such a mechanism is to ensure that the nature of the support does not influence the previously mentioned steady-state operation of the mechanism, while 


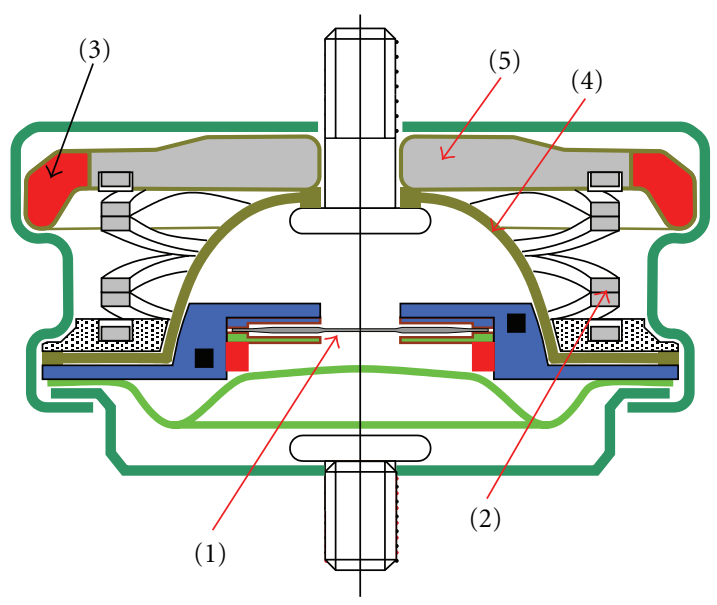

Figure 3: Proposed hydraulic engine mount design.

still maintaining the advantage of the supported decoupler in the initial transient response. Here the decoupler disk is supported and forced to be in neutral position equidistance between either cage limit; however, the decoupler is not fixed from motion. This design requires the decoupler to be made of an elastomeric material to provide sufficient stiffness to keep the decoupler located during nonexcited (static) situations, and provide sufficient flexibility to allow normal operation during dynamic events.

Up to the present, very few researchers have looked at the start-up or transient behavior of the hydraulic mount with Adiguna et al. being among the few [11]. However, the behavior of the mount for a bottomed-up decoupler has never been investigated, although after a short period of time the decoupler recovers its purposed function and does what it is designed for. However, in the current floating-decoupler design the decoupler might simply sit against one of the cage bounds while not excited, depending upon mounting configurations and density mismatch with the surrounding fluid, therefore causing the system to initially utilize only the inertia track. Therefore, after every excitation removal the decoupler might sink or float to create the problem again. With either condition it becomes apparent, after some consideration, that because it is the decoupler that allows the mount to act as either a low damping or a high damping mechanism by means of its position, then the position of the decoupler during the aforementioned excitations is quite important.

\section{Suspended Decoupler HEM Model Description}

Floating decoupler HEM is described in the literature very well [5-16]. To be compared with float type, here we describe a suspended decoupler HEM. Noting the disadvantages of the floating decoupler compared to suspended decoupler mount, it seems advantageous to design a new mount utilizing such a suspended decoupler mechanism. Such a mount should provide effective isolation characteristics through a broad frequency spectrum while maintaining or surpassing existing hydraulic engine mount benchmarks for performance.

Figure 3 illustrates a schematic of the proposed design intended to meet the aforementioned criteria. The mount utilizes the same decoupler mechanism (1) as illustrated in Figure 2. In addition, the mount does away with the traditional upper rubber structure common to practically every modern hydraulic engine mount. Instead the proposed mount makes use of a Belleville spring (2) to provide the primary axial stiffness and a thick circumferential rubber band (3) surrounding the upper structure of the mount to limit transverse motions of the mount. The volumetric compliance of the upper chamber of the mount is provided through a relatively thick rubber chamber (4) which is mechanically fastened to the upper moving head of the engine mount (5). The advantage of such a structure over the traditional rubber structure is twofold. First, the stiffness of the engine mount is more tunable and is as simple as appropriate spring sizing as compared to complicated geometrical designs required for the current rubber structure. Second, the damping of the system can be allowed to rest with the fluid motion inside of the mount thereby allowing more precise tuning by means of inertia track and decoupler geometry [10]. Such a method is far simpler than trying to design an upper rubber structure with a specified amount of hysteretic-type damping.

Figure 4 illustrates the three-dimensional representation of the design. Here the decoupler geometry becomes clearer in conjunction with the design of the upper structure. Figure 5 provides a better illustration of the decoupler geometry required to achieve the aforementioned requirements. As illustrated in Figure 5 the decoupler support tabs are thinned regions with slots on either side to help them to not dramatically influence overall decoupler dynamics while still maintaining the required stiffness to ensure proper decoupler position during static conditions.

\section{Dynamic Parameters Evaluations}

In every HEM there are two rubber-type components in upper and lower chambers to collect the moving fluid. These rubbery components produce compliances of the system which appear in the equations of motion. Besides the two chambers, the suspended decoupler also show an elastic behavior. Utilizing FEM we show how to determine the elastic behavior of the decoupler, upper bellow, and lower collector compliances.

To begin the analysis of the engine mount it is paramount that the necessary geometric and material parameters are identified. To accomplish such, finite element analysis is utilized as a tool to provide knowledge of component loaddeflection relationships, volumetric expansion properties, and so forth. By creating a finite element model based upon the geometry illustrated in Figure 5, information regarding the load-deflection behavior of the decoupler mechanism is readily obtainable. Figure 6 illustrates the discretized finite element model. The model was discretized using 10 node tetrahedral elements with a total of 42,794 active degrees of freedom for the model. 

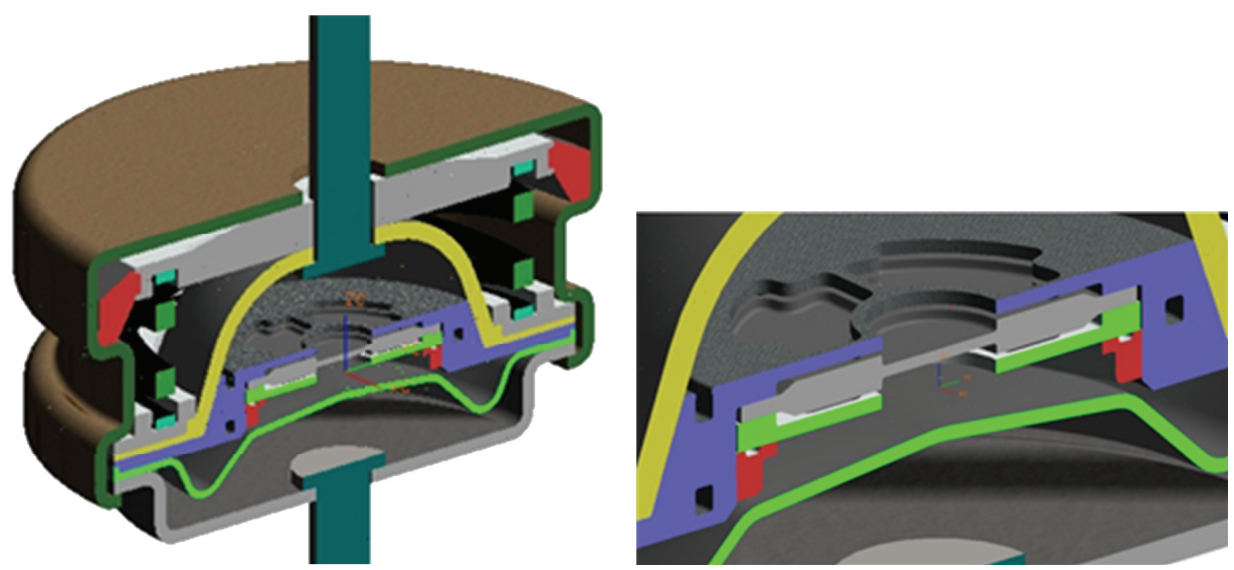

FIgURE 4: Model of the proposed hydraulic mount.

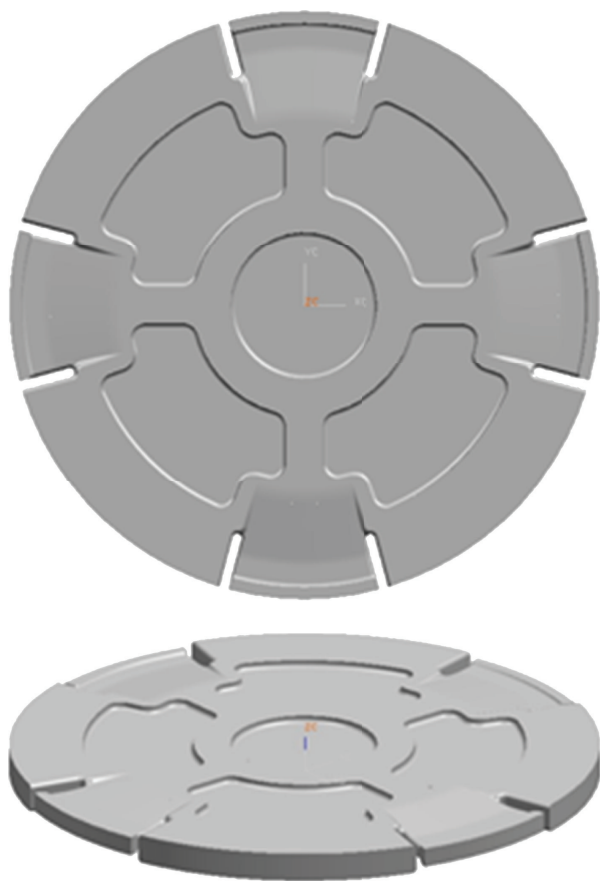

(a) Decoupler plate

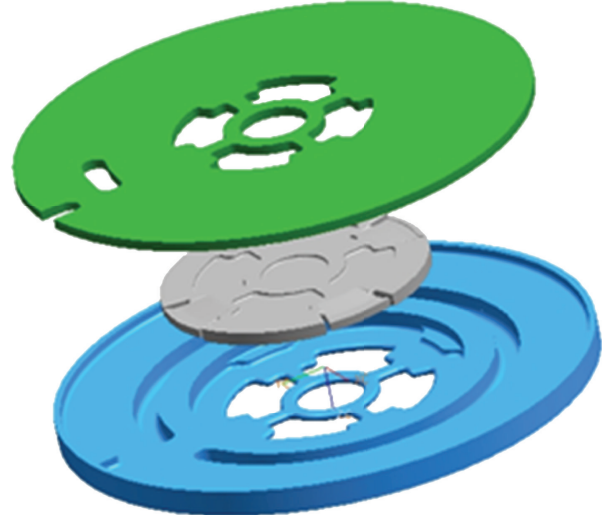

(b) Housing of decoupler plate

Figure 5: Decoupler geometry.

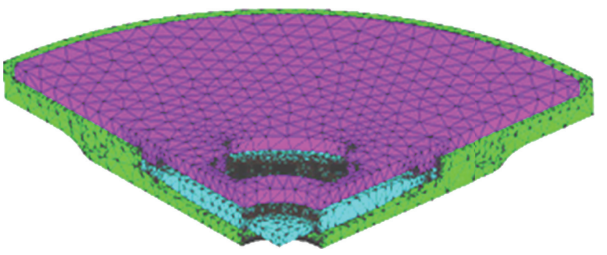

Figure 6: Discretized finite element model.

To simulate the impact condition between the decoupler and the surrounding cage bounds Lagrangian typecontact elements were imposed upon potential impacting surfaces (see Figure 7). The contact region at the decoupler support points was simulated using a rough-style interface between the two materials thereby allowing no slippage [17]. Whereas the surfaces contacting after sufficient decoupler deformation were treated as frictionless thereby allowing relative motion between the two bodies. To simplify the analysis and determine the effectiveness of the new design compared to floating decoupler design, we ignore the fluidsolid interaction as is done in modeling HEM [8-16].

Because the decoupler is to be made of an elastomeric material the three-parameter Mooney-Rivlin model, illustrated in (1) is utilized [18-20]. The three-parameter Mooney-Rivlin model expresses the strain energy density as 


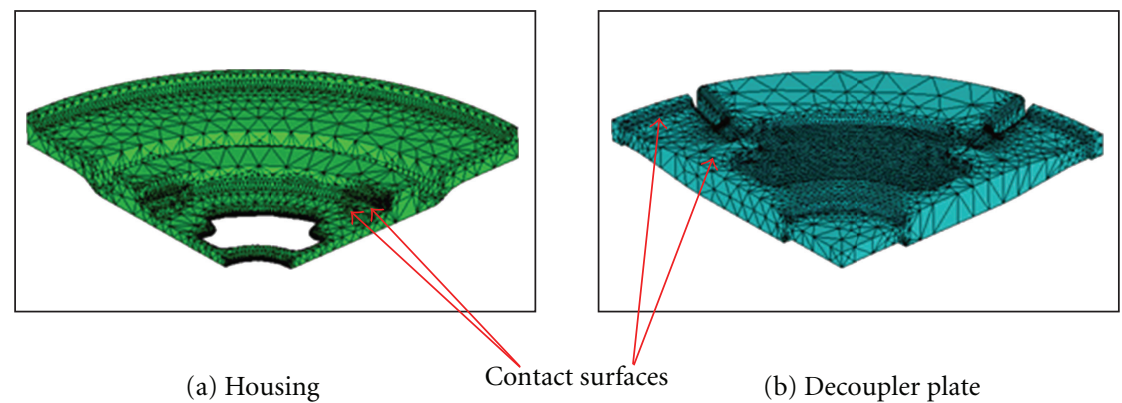

FIGURE 7: Contact element surfaces (not in scale).

Table 1: Mooney-Rivlin constants.

\begin{tabular}{ll}
\hline Parameter & Value $(\mathrm{MPa})$ \\
\hline$c_{10}$ & $4.838 \mathrm{E}-01$ \\
$c_{01}$ & $-9.456 \mathrm{E}-02$ \\
$c_{11}$ & $1.235 \mathrm{E}-02$ \\
\hline
\end{tabular}

a function of the material constants $\left(c_{10}, c_{01}\right.$, and $\left.c_{11}\right)$, and the first two invariants $\left(I_{1}\right.$ and $\left.I_{2}\right)$ of the Right CauchyGreen deformation tensor [19-21]. The material constants that we adapted are shown in Table 1, and may be obtained from experiment by means of a least-squares curve-fitting procedure $[15,22,23]$,

$$
W=c_{10}\left(I_{1}-3\right)+c_{01}\left(I_{2}-3\right)+c_{11}\left(I_{1}-3\right)\left(I_{2}-3\right) .
$$

To solve the finite element model an applied numerical solution method must be employed. Such an approach was required due primarily to two factors. First, the material for the decoupler is nonlinear and requires full geometric nonlinearity options to be utilized. Second, the contact between the rubber and metallic cage bounds is asymmetric noting the differences in material responses between the two structures; therefore, the full Newton-Raphson approach must be employed to deal with the unsymmetrical nature of the assembled matrices [17].

The fluid is assumed to be incompressible compared to elastic and flexible parts. To simulate fluid-induced pressure an evenly distributed pressure of $20 \mathrm{kPa}$ was assumed to one side of the entire exposed surface of the decoupler. To constrain the entire assembly from motion the lower surface of the cage was fixed in all degrees of freedom. In order to obtain information regarding the load-deflection relationship of the supported decoupler the applied pressure was resolved into a force component by multiplying the area upon which the pressure was applied. The corresponding deflection measurement was taken in the vertical direction from the center node (exposed due to symmetry conditions) of the decoupler disk. The results of the finite element analysis are illustrated in Figure 8.

Notice from Figure 8 that even after the decoupler impacts the cage bounds the disk, it continues to displace with a corresponding increase in applied load due to the elastic nature of the decoupler material. In addition, notice that

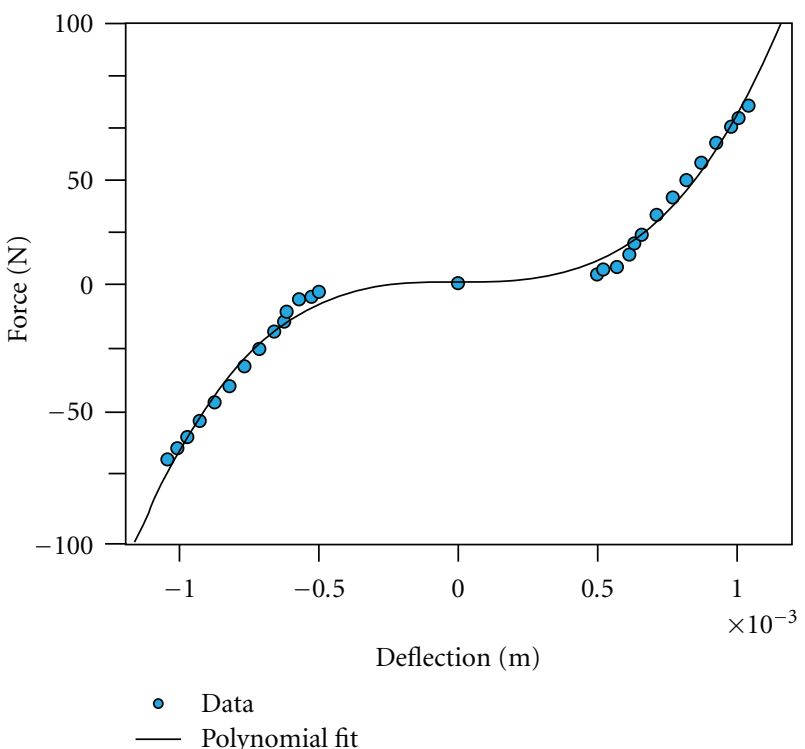

FIGURE 8: Decoupler load-deflection relationship.

TABLE 2: Material properties.

\begin{tabular}{lcc}
\hline Component & Young's modulus $(\mathrm{GPa})$ & Poisson's ratio \\
\hline Spring & 207 & 0.30 \\
Upper structure & 71 & 0.33 \\
Spring support & 71 & 0.33 \\
\hline
\end{tabular}

a third-order polynomial, expressed in (2), approximates the data with reasonably good accuracy with $E_{1}=8.0246 \mathrm{~N}$,

$$
f_{d}\left(x_{d}, \Delta\right)=E_{1} \frac{x_{d}^{3}}{\Delta^{3}} .
$$

Consider the upper structure of the engine mount with material properties shown in Table 2. The structure is considered as a whole because of the nonlinearity inherent in the load-deflection relationship of the spring, but also the material nonlinearity of the rubber components. Because of the nonlinearity, the principle of superposition is not applicable; therefore, the stiffness of the upper structure will be modeled by one nonlinear spring element (as compared to multiple springs in parallel). Figure 9 illustrates the model geometry and corresponding finite element mesh 


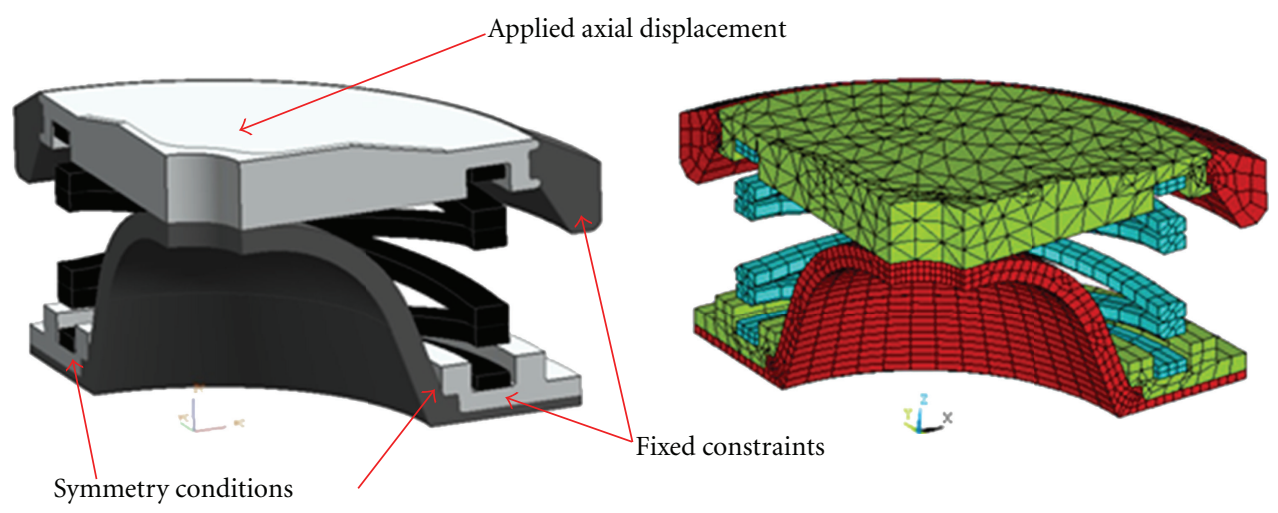

FIgURE 9: Upper structure model and meshed geometry.

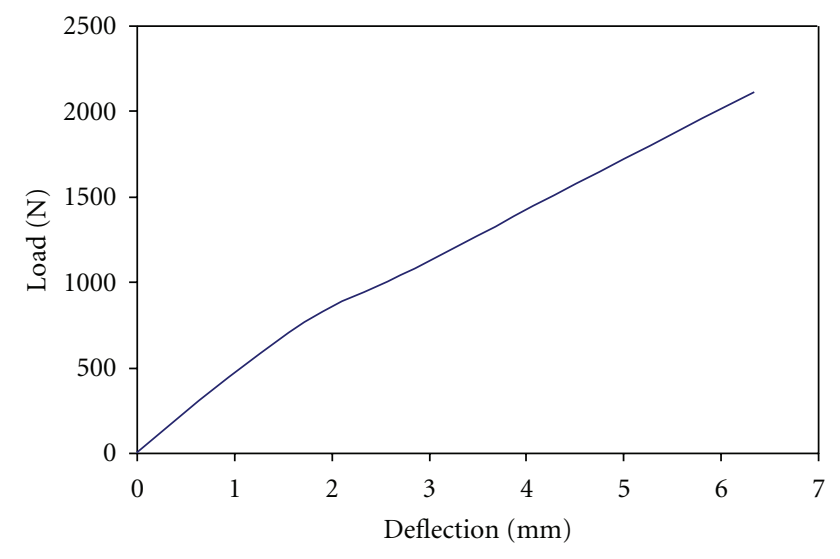

FIGURE 10: Load-deflection relationship.

which consisted of 20 node hexahedral elements and 10 node tetrahedral elements with a total of 71,211 degrees of freedom. Additionally, contact surfaces were specified everywhere metallic components are in contact or were to contact. Bonded-type contact surfaces were specified everywhere that elastomeric materials were in contact with metallic components as the design intent was to have said metallic components bonded to the rubber parts as a part of the manufacturing process.

The finite element model was constrained on the lower surface with load being applied in the form of a specified displacement in the axial direction on the opposing surface. In addition, fixed constraints were applied to the lower and outer surfaces of the surrounding rubber component as illustrated in Figure 9.

To solve the finite element model a nonlinear simulation was utilized allowing for finite strains. Figure 10 illustrates the resulting load-deflection relationship obtained from the finite element analysis. Because of nonlinearity, a thirddegree polynomial is fit to the data. Equation (3) is the result of a least-squares curve fit to the finite element results. In this equation, the input deflection $x$ has units of $\mathrm{mm}$,

$$
F=5.2605 x^{3}-63.907 x^{2}+526.73 x
$$

Next, consider the upper bellows and its corresponding volumetric compliance. The corresponding finite element model is illustrated in Figure 11. The mesh consisted of 8 node quadrilateral-type elements with 768 total degrees of freedom. The analysis allowed for finite strains to account for the hyperelastic behavior of the rubber upper compliance, and therefore required solution by the NewtonRaphson approach. The finite element model illustrated in Figure 11 was constrained from motion on the bottom and top surfaces while an evenly distributed pressure was applied on the internal surface of the upper compliance to simulate fluid pressure.

Figure 12 illustrates the volume-pressure relationship for the upper bellows structure. Note the relative linearity of the relationship; therefore, by using a least-squares fit of a linear line to the data results in a line with a slope of $2.457 \mathrm{E}-$ $09 \mathrm{~m}^{5} / \mathrm{N}$, which corresponds to the volumetric compliance of the upper bellows structure.

Determination of the lower chamber volumetric compliance is accomplished much the same as for the upper chamber. Figure 13 illustrates the finite element model for the lower chamber. However, to model the rubber compliance shell elements were utilized noting the constant thickness of the part, and the large deformations this structure is intended to undergo. In addition, due to the large deformations expected the rubber compliance has the tendency to buckle outwards. This buckling is difficult to model using solid hexahedral elements noting such deformations can result in unacceptable element shapes and potentially inaccurate solutions; therefore, 4 node shell elements were employed as such deformations do not necessarily cause such element shape problems $[24,25]$. Figure 14 illustrates the results of the analysis of the model illustrated in Figure 13.

Notice the behavior of the lower compliance illustrated in Figure 14 is also nonlinear; however, it appears approximately bilinear. After closer investigation the initial portion of the volume-pressure curve represents the chambers initial expansion until it contacts the surrounding structural walls. At the point where contact between the two bodies initiates the slope of the volume-pressure curve drastically changes indicating a less compliant structure. It is the slope of this segment of line that is used to approximate the volumetric compliance of the lower structure noting the small amount 

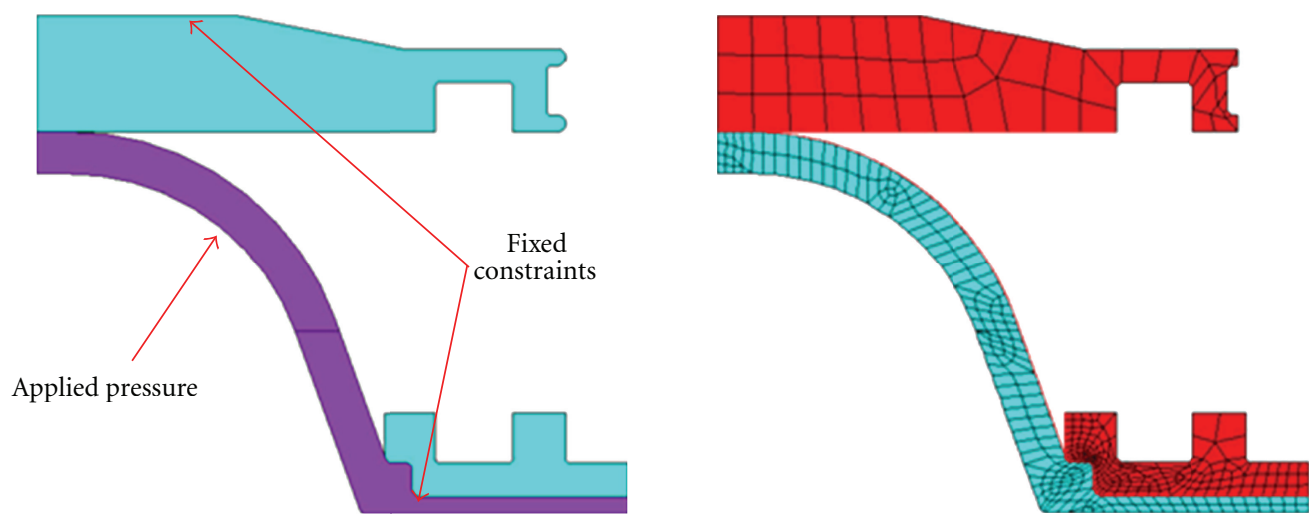

FIGURE 11: Upper compliance model and meshed geometry.

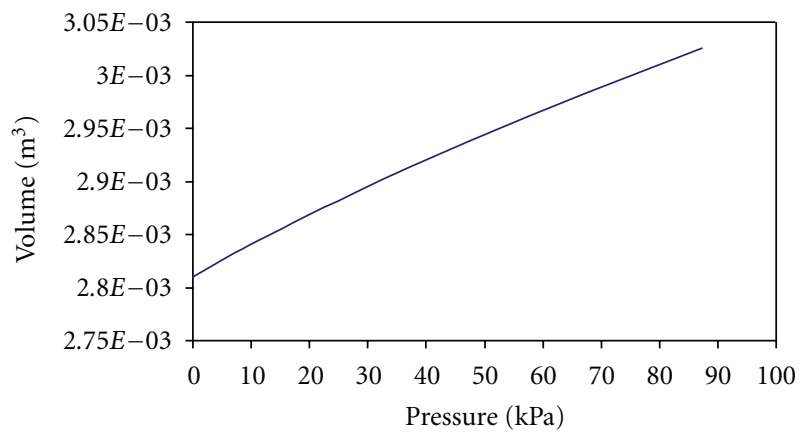

FIgURE 12: Volume-pressure relationship (upper compliance).

TABLE 3: Hydraulic mount parameters.

\begin{tabular}{lcc}
\hline Property & Value & Unit \\
\hline$A_{d}$ & $8.107 \mathrm{E}-05$ & $\mathrm{~m}^{2}$ \\
$A_{i}$ & $5.026 \mathrm{E}-06$ & $\mathrm{~m}^{2}$ \\
$A_{p}$ & $3.663 \mathrm{E}-03$ & $\mathrm{~m}^{2}$ \\
$B_{d}$ & $1.031 \mathrm{E}-04$ & $\mathrm{Ns} / \mathrm{m}$ \\
$B_{i}$ & 3.257 & $\mathrm{Ns} / \mathrm{m}$ \\
$B_{r}$ & $0.50 \mathrm{E}+02$ & $\mathrm{Ns} / \mathrm{m}$ \\
$C_{1}$ & $2.457 \mathrm{E}-09$ & $\mathrm{~m}^{5} / \mathrm{N}$ \\
$C_{2}$ & $2.674 \mathrm{E}-09$ & $\mathrm{~m}^{5} / \mathrm{N}$ \\
$k_{1}$ & 5.2605 & $\mathrm{~N} / \mathrm{mm}^{3}$ \\
$k_{2}$ & -32.344 & $\mathrm{~N} / \mathrm{mm}^{2}$ \\
$k_{3}$ & 334.22 & $\mathrm{~N} / \mathrm{mm}^{2}$ \\
$M_{d}$ & $5.220 \mathrm{E}-04$ & $\mathrm{~kg}$ \\
$M_{i}$ & $1.354 \mathrm{E}-03$ & $\mathrm{~kg}$ \\
$E$ & 0.50 & - \\
$E_{1}$ & 8.0246 & $\mathrm{~N}$ \\
$\Delta$ & 0.5 & $\mathrm{~mm}$ \\
$Y$ & 1.0 & $\mathrm{~mm}$ \\
\hline
\end{tabular}

of fluid pressure required to move the system operating point into this region. Such an assumption in regards to the system operating point being located in said region can be validated by noting that the static load of the engine is sufficient to cause such an operating point shift. Table 3 introduces the complete compliment of hydraulic engine mount parameters [10].

\section{Mathematical Analysis}

By introducing the support to the decoupler the momentum balance equation for the decoupler exhibits a restoring force term. Additionally, the nonlinear damping term first introduced by Golnaraghi and Jazar is utilized [13, 14]. However, to fully describe system dynamics, the inertia track momentum equation is needed along with the fluid continuity equations $[10-14,26,27]$. In this commonly accepted modeling, the fluid-solid interaction is ignored,

$$
\begin{gathered}
M_{d} \ddot{x}_{d}+\left(B_{d}+E \frac{x_{d}^{2}}{\Delta^{2}}\right) \dot{x}_{d}+f_{d}\left(x_{d}, \Delta\right)=A_{d}\left(P_{1}-P_{2}\right), \\
M_{i} \ddot{x}_{i}+B_{i} \dot{x}_{i}=A_{i}\left(P_{1}-P_{2}\right), \\
A_{p}(\dot{x}-\dot{y})=A_{i} \dot{x}_{i}+A_{d} \dot{x}_{d}+C_{1}\left(\dot{P}_{1}-\dot{P}_{\mathrm{atm}}\right), \\
A_{d} \dot{x}_{d}+A_{i} \dot{x}_{i}=C_{2}\left(\dot{P}_{2}-\dot{P}_{\mathrm{atm}}\right) .
\end{gathered}
$$

Equations (4) and (5) are momentum balance of the fluid mass in decoupler canal and inertia track, while the (5) and (6) are continuity equations for upper and lower chambers, respectively. Utilizing (4) through (7) results in the following equations of motion which describe the internal dynamics of the hydraulic mount:

$$
M \ddot{q}+C \dot{q}+K q+f_{d}=\mathbf{f},
$$

where,

$$
\begin{gathered}
\mathbf{M}=\left[\begin{array}{cc}
M_{d} & 0 \\
0 & M_{i}
\end{array}\right], \quad \mathbf{C}=\left[\begin{array}{cc}
B_{d}+E \frac{x_{d}^{2}}{\Delta^{2}} & 0 \\
0 & B_{i}
\end{array}\right], \\
\mathbf{K}=\left[\begin{array}{cc}
A_{d}^{2} K & A_{d} A_{i} K \\
A_{d} A_{i} K & A_{i}^{2} K
\end{array}\right],
\end{gathered}
$$



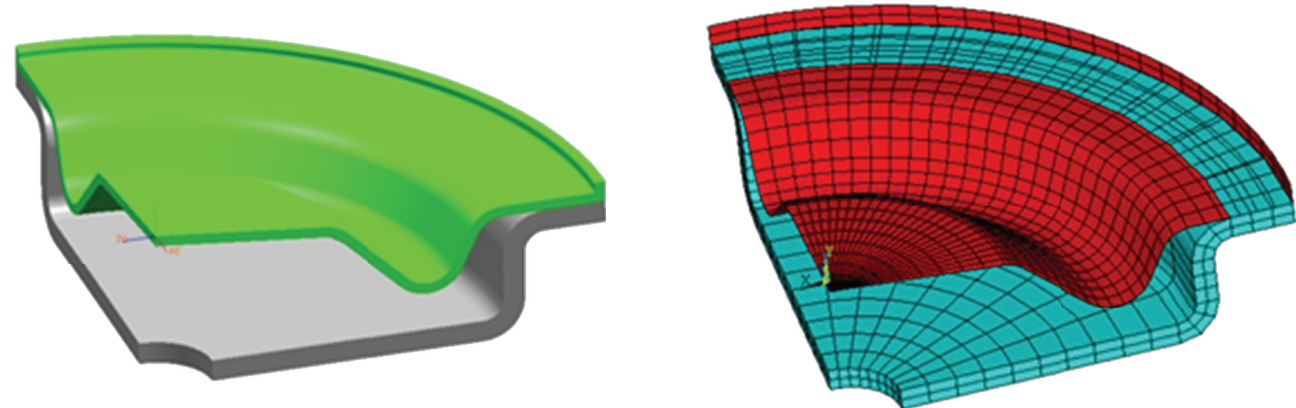

FIGURE 13: Lower compliance model and meshed geometry.

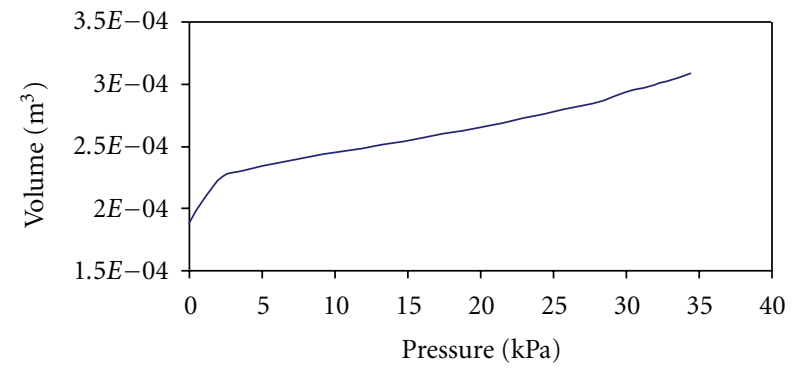

FIGURE 14: Volume-pressure relationship (lower compliance).

$$
\begin{gathered}
\mathbf{f}_{\mathbf{d}}=f_{d}\left(x_{d}, \Delta\right)\left\{\begin{array}{l}
1 \\
0
\end{array}\right\}, \quad \mathbf{f}=\frac{A_{p} X}{C_{1}}\left\{\begin{array}{l}
A_{d} \\
A_{i}
\end{array}\right\} \sin (\omega t), \quad \mathbf{q}=\left\{\begin{array}{c}
x_{d} \\
x_{i}
\end{array}\right\}, \\
K=C_{1}^{-1}+C_{2}^{-1} .
\end{gathered}
$$

In order to make the analysis general, the following nondimensional parameters are introduced:

$$
\begin{gathered}
\tau=\Omega t, \quad \Omega^{2}=\frac{A_{d}^{2} K}{M_{d}}, \quad x_{d}=\Delta y_{d}, \\
x_{i}=\Delta y_{i}, \quad x=\Delta y .
\end{gathered}
$$

Using the parameters in (10), (8) is now expressed in the following nondimensional forms:

$$
\begin{gathered}
y_{d}^{\prime \prime}+\left(\zeta_{d}+e y_{d}^{2}\right) y_{d}^{\prime}+g_{d}\left(y_{d}\right)+y_{d}+a y_{i}=f Y \sin (w \tau), \\
y_{i}^{\prime \prime}+\zeta_{i} y_{i}^{\prime}+\frac{a}{m} y_{d}+\frac{a^{2}}{m} y_{i}=\frac{f a}{m} Y \sin (w \tau),
\end{gathered}
$$

where,

$$
\begin{gathered}
w=\frac{\omega}{\Omega}, \quad a=\frac{A_{i}}{A_{d}}, \quad m=\frac{M_{i}}{M_{d}}, \quad f=\frac{A_{p}}{C_{1} K A_{d}}, \\
e=\frac{E}{M_{d} \Omega}, \quad \zeta_{d}=\frac{B_{d}}{M_{d} \Omega}, \quad \zeta_{i}=\frac{B_{i}}{M_{i} \Omega}, \\
g_{d}\left(y_{d}\right)=\frac{f_{d}\left(x_{d}, \Delta\right)}{\Delta \Omega^{2} M_{d}} .
\end{gathered}
$$

Introducing the small parameter $\boldsymbol{\varepsilon}$ as a measure of the nonlinearity, the following nondimensional parameters are instituted:

$$
\begin{aligned}
\varepsilon=a, & \varepsilon d_{d}=\zeta_{d}, & \varepsilon d_{i}=\zeta_{i}, \\
\varepsilon q=e, & \varepsilon g=f Y, & \nu=\frac{a}{m} .
\end{aligned}
$$

Using the parameters in (14) the equations of motion from (11) and (12) are now expressed:

$$
\begin{gathered}
y_{d}^{\prime \prime}+\varepsilon\left(d_{d}+q y_{d}^{2}\right) y_{d}^{\prime}+g_{d}+y_{d}+\varepsilon y_{i}=\varepsilon g \sin (w \tau), \\
y_{i}^{\prime \prime}+\varepsilon d_{i} y_{i}^{\prime}+v y_{d}+\varepsilon v y_{i}=\varepsilon v g \sin (w \tau) .
\end{gathered}
$$

To obtain a solution in the frequency domain for (15) the averaging method is employed by introducing an assumed solutions in the following form $[28,29]$ :

$$
\begin{gathered}
y_{d}=r_{d}(\tau) \sin \left(\tau+\phi_{d}(\tau)\right), \\
y_{i}=r_{i}(\tau) \sin \left(\tau+\phi_{i}(\tau)\right), \\
y_{d}^{\prime}=r_{d}(\tau) \cos \left(\tau+\phi_{d}(\tau)\right), \\
y_{i}^{\prime}=r_{i}(\tau) \cos \left(\tau+\phi_{i}(\tau)\right) .
\end{gathered}
$$

Expressing the first derivatives as in (18) and (19) requires two constraint equations to maintain the validity of the solution,

$$
\begin{gathered}
r_{d}^{\prime}(\tau) \sin \left(\tau+\phi_{d}(\tau)\right)+r_{d} \phi_{d}^{\prime}(\tau) \cos \left(\tau+\phi_{d}(\tau)\right)=0, \\
r_{i}^{\prime}(\tau) \sin \left(\tau+\phi_{i}(\tau)\right)+r_{i} \phi_{i}^{\prime}(\tau) \cos \left(\tau+\phi_{i}(\tau)\right)=0 .
\end{gathered}
$$

Now the second-time derivatives can be obtained directly from (18) and (19):

$$
\begin{aligned}
y_{d}^{\prime \prime}= & r_{d}^{\prime}(\tau) \cos \left(\tau+\phi_{d}(\tau)\right)-r_{d}(\tau)\left(1+\phi_{d}^{\prime}(\tau)\right) \\
& \times \sin \left(\tau+\phi_{d}(\tau)\right), \\
y_{i}^{\prime \prime}= & r_{i}^{\prime}(\tau) \cos \left(\tau+\phi_{i}(\tau)\right)-r_{i}(\tau)\left(1+\phi_{i}^{\prime}(\tau)\right) \\
& \times \sin \left(\tau+\phi_{i}(\tau)\right) .
\end{aligned}
$$


Equations (16) through (19) and (21) are now substituted directly into the equations of motion and utilized in conjunction with (20) to transform the second-order differential equations in (15) into a system of four first-order differential equations. After extracting the slow terms of the resulting first-order differential equations, averaging over one period of oscillation, the equations of motion in terms of the firstorder differential equations are obtained:

$$
\begin{gathered}
r_{d}=-\frac{\varepsilon}{2}\left(g \sin \left(w \tau-\tau-\phi_{d}\right)+d_{d} r_{d}\right), \\
r_{d} \phi_{d}^{\prime}=\frac{\varepsilon}{2}\left(s r_{d}^{3}-g \cos \left(w \tau-\tau-\phi_{d}\right)+\nu r_{d}+\frac{q r_{d}^{3}}{4}\right), \\
r_{i}^{\prime}=-\frac{\varepsilon}{2}\left(d_{i} r_{i}+\nu g \sin \left(w \tau-\tau-\phi_{i}\right)\right), \\
r_{i} \phi_{i}^{\prime}=\frac{\varepsilon \nu}{2}\left(g \cos \left(w \tau-\tau-\phi_{i}\right)+r_{i}\right),
\end{gathered}
$$

where,

$$
s=\frac{E_{1}}{A_{d}^{2} K \Delta} .
$$

In order for equations (22) through (25) to be useable in a frequency domain consider the following transformation to allow conversion of (22) through (25) to an autonomous system of equations:

$$
\begin{gathered}
w \tau-\tau-\phi_{d}=\psi_{d} \\
w \tau-\tau-\phi_{i}=\psi_{i} .
\end{gathered}
$$

Utilizing (27) in equations (22) through (25) and noting that for steady-state conditions to prevail the time derivatives must vanish results in implicit frequency response functions for the system,

$$
\begin{gathered}
\left(\frac{d_{d} r_{d}}{g}\right)^{2}+\left(\frac{r_{d}\left(4 \varepsilon s r_{d}^{2}+8+4 \varepsilon v+\varepsilon q r_{d}^{2}-8 w\right)}{4 \varepsilon g}\right)^{2}=1, \\
\left(\frac{d_{i} r_{i}}{\nu g}\right)^{2}+\left(\frac{r_{i}(\varepsilon v-2 w+2)}{\varepsilon v g}\right)^{2}=1 .
\end{gathered}
$$

Equations (28) are identical to the frequency response functions obtained in $[13,16]$ for a floating decoupler mount if $s$ is allowed to equal zero thereby validating the solution noting that the only difference mathematically between the two systems is the $s y_{d}^{3}$ term.

\section{Dynamic Responses}

Figure 15 illustrates the frequency response function for both the supported decoupler introduced in this investigation and the unsupported decoupler model from [10]. It is seen there is no discernible difference between the two models indicating that by supporting the decoupler disk the overall function of the mechanism was not substantially affected in its steady-state response.

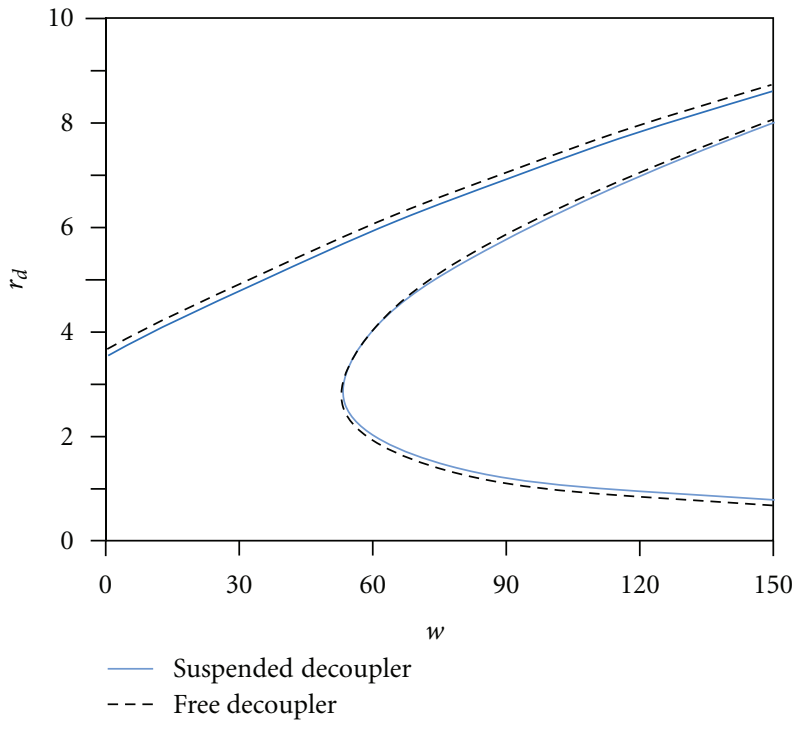

FIGURE 15: Decoupler frequency response.

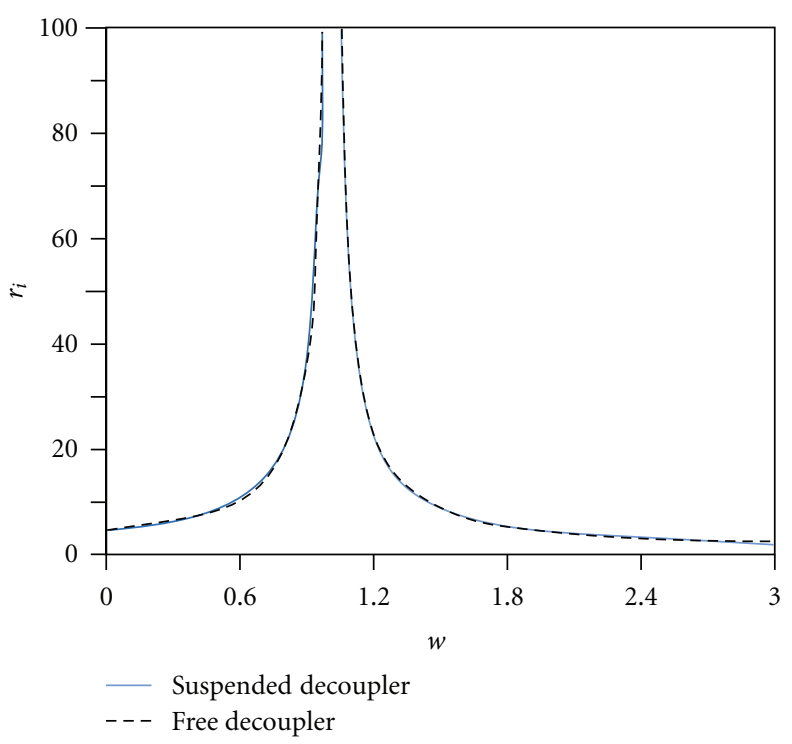

FIGURE 16: Inertia track frequency response.

Figure 16 illustrates the inertia track frequency response function for the mount obtained from the averaging solution above in conjunction with the solution from [10] indicating no appreciable difference or effect on its behavior due to the decoupler modification.

Noting that the supported decoupler design is based on the initial transient response of the system, consider the force transmitted through the engine mount due to a $1 \mathrm{~mm}$ pulse input held for a period of 0.1 seconds. To calculate the force transmitted through the engine mount, consider the following equation developed in [14] and illustrated here to describe the response to a step input. The transmitted force 


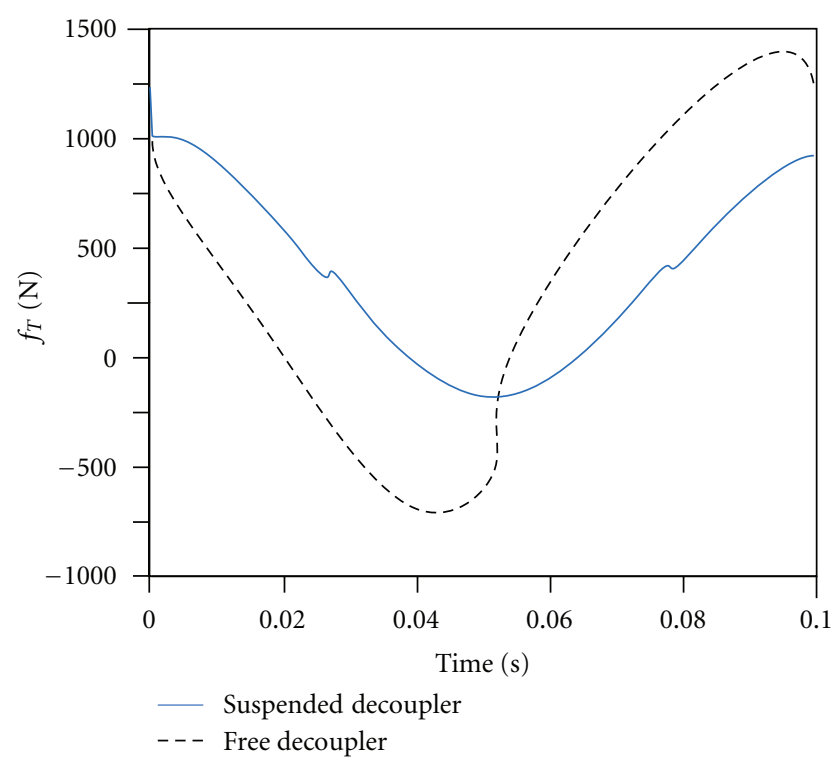

Figure 17: Transmitted force.

is the mount dynamic including the nonlinear stiffness of the upper rubber,

$$
f_{T}(t)=k_{1} x(t)^{3}+k_{2} x(t)^{2}+k_{3} x(t)+B_{r} \dot{x}(t)+A_{p} P_{1}(t) .
$$

Determining the solution to the equations of motion in (8) numerically allows determination of the pressure term in (29) by means of numerical integration of the continuity equations, thereby allowing determination of the transmitted force by means of (29).

Figure 17 illustrates the force transmitted through the engine mount for the supported decoupler mount and the free decoupler mount. In this analysis we assumed the initial condition of the floating decoupler to be bottomup position. Therefore, the supported decoupler mount transmits substantially less force $(\sim 200 \mathrm{~N})$ at startup as compared to the free decoupler mount. In addition, the maximum amplitude of force transmitted via the supported decoupler mount is $716 \mathrm{~N}$ whereas the maximum amplitude of force transmitted via the free decoupler mount is $1.060 \mathrm{E}+$ $03 \mathrm{~N}$. The supported decoupler mount provides a reduction in peak amplitude on startup of $32.5 \%$ over the free decoupler mount thereby indicating the effectiveness of the supported decoupler design. In addition, Figures 16 and 17 illustrate that by utilizing the supported decoupler the steady-state dynamics of the engine mount are not effected in a measurable amount; therefore, the supported decoupler design has been shown to be superior in improving overall system dynamics and mount isolation characteristics.

\section{Conclusion}

This study has introduced a decoupler design motivated by the desire to improve upon the current floating-decoupler design. Using nonlinear finite elements, information in regards to the structural elastic behavior was obtained.
This information was then readily utilized by the lumped parameter modeling approach utilized by practically all researchers investigating hydraulic engine mounts. Using the lumped parameter model, the frequency response of the system was investigated utilizing averaging method and compared to previously published results describing floating-decoupler-type mounts with excellent agreement. The agreement between the two models indicated that by supporting the decoupler on thin, low-stiffness tabs, the overall steady-state response of the system is practically unaffected. Additionally, by using numerical analysis to determine the transient response of the system, the supported decoupler substantially improves the engine mounts' response to sudden excitations. Future work must be about optimizing the supported decoupler design illustrated in this investigation utilizing the RMS optimization method.

\section{Abbreviations}

$\begin{array}{ll}A: & \text { Area } \\ B: & \text { Equivalent viscous damping coefficient } \\ C: & \text { Volumetric compliance } \\ E: & \text { Nonlinear decoupler damping } \\ E_{1}: & \text { Coefficient } \\ f: & \text { Nonlinear decoupler force coefficient } \\ K=1 / C_{1}+1 / C_{2}: & \text { Inverse sum of compliances } \\ k_{1}: & \text { Upper rubber load-deflection } \\ & \text { Coefficient } \\ k_{2}: & \text { Upper rubber load-deflection } \\ & \text { coefficient } \\ k_{3}: & \text { Upper rubber equivalent stiffness } \\ M: & \text { Mass } \\ P: & \text { Pressure } \\ Q: & \text { Flow rate } \\ R: & \text { RMS of acceleration transmissibility } \\ t: & \text { Time } \\ x: & \text { Position } \\ y: & \text { Excitation } \\ \Delta: & \text { Gap size } \\ \omega: & \text { Excitation frequency } \\ \xi: & \text { Damping ratio } \\ \omega_{n}: & \text { Natural frequency } \\ r: & \text { Nondimensional amplitude } \\ w: & \text { Nondimensional frequency } \\ I_{1}, I_{2}: & \text { Tensor invariants } \\ c_{10}, c_{01}, c_{11}: & \text { Material constants } \\ W: & \end{array}$

\section{Subscripts}

$\begin{array}{ll}i: & \text { Inertia track } \\ d: & \text { Decoupler } \\ p: & \text { Piston } \\ r: & \text { Rubber } \\ 1: & \text { Upper chamber } \\ 2: & \text { Lower chamber } \\ \text { atm: } & \text { Atmosphere } \\ T: & \text { Transmitted }\end{array}$




\section{References}

[1] W. C. Flower, "Understanding hydraulic mounts for improved vehicle noise, vibration and ride qualities," SAE Technical Paper Series 850975, 1985.

[2] M. Bernuchon, "A new generation of engine mounts," SAE Technical Paper Series 840259, 1984.

[3] J. C. Snowdon, "Vibration isolation: use and characterization," Journal Acoustic Society of America, vol. 66, no. 5, pp. 12451274, 1979.

[4] J. P. Den Hartog, Mechanical Vibrations, McGraw Hill, New York , NY, USA, 5th edition, 1956.

[5] R. Singh, G. Kim, and P. V. Ravindra, "Linear analysis of automotive hydro-mechanical mount with emphasis on decoupler characteristics," Journal of Sound and Vibration, vol. 158, no. 2, pp. 219-243, 1992.

[6] M. Clark, "Hydraulic engine mount isolation," SAE Technical Paper Series 851650, 1986.

[7] M. Sugino and E. Abe, "Optimum application for hydroelastic engine mount," SAE Technical Paper Series 861412, 1986.

[8] K. H. Lee, Y. T. Choi, and S. P. Hong, "Performance analysis of hydraulic engine mount by using bond graph method," SAE Technical Paper Series 951347, 1995.

[9] P. E. Corcoran and G. H. Ticks, "Performance analysis of hydraulic engine mount by using bond graph method," SAE Technical Paper Series 840407, 1984.

[10] J. Christopherson and G. N. Jazar, "Optimization of classical hydraulic engine mounts based on RMS method," The Shock and Vibration Digest, vol. 12, no. 2, pp. 119-147, 2005.

[11] H. Adiguna, M. Tiwari, R. Singh, H. E. Tseng, and D. Hrovat, "Transient response of a hydraulic engine mount," Journal of Sound and Vibration, vol. 268, no. 2, pp. 217-248, 2003.

[12] M. Tiwari, H. Adiguna, and R. Singh, "Experimental characterization of a nonlinear hydraulic engine mount," Noise Control Engineering Journal, vol. 51, no. 1, pp. 36-49, 2003.

[13] M. F. Golnaraghi and R. N. Jazar, "Development and analysis of a simplified nonlinear model of a hydraulic engine mount," Journal of Vibration and Control, vol. 7, no. 4, pp. 495-526, 2001.

[14] R. N. Jazar and M. F. Golnaraghi, "Nonlinear modeling, experimental verification, and theoretical analysis of a hydraulic engine mount," JVC/Journal of Vibration and Control, vol. 8, no. 1, pp. 87-116, 2002.

[15] J. Christopherson and R. N. Jazar, "Dynamic behavior comparison of passive hydraulic engine mounts. Part 2: finite element analysis," Journal of Sound and Vibration, vol. 290, no. 3-5, pp. 1071-1090, 2006.

[16] J. Christopherson and R. N. Jazar, "Dynamic behavior comparison of passive hydraulic engine mounts. Part 1: mathematical analysis," Journal of Sound and Vibration, vol. 290, no. 3-5, pp. 1040-1070, 2006.

[17] SAS IP, Inc., ANSYS 8.0 Help Documentation, 2003.

[18] T. Belytschko, W. K. Liu, and B. Moran, Nonlinear Finite Elements for Continua and Structures, John Wiley \& Sons, New York, NY, USA, 2000.

[19] D. J. Charlton, Y. Yang, and K. K. Teh, "Review of methods to characterize rubber elastic behavior for use in finite element analysis," Rubber Chemistry and Technology, vol. 67, no. 3, pp. 481-503, 1994.

[20] D. J. Seibert and N. Schocke, "Direct comparison of some recent rubber elasticity models," Rubber Chemistry and Technology, vol. 73, no. 2, pp. 366-384, 2000.
[21] M. M. Attard and G. W. Hunt, "Hyperelastic constitutive modeling under finite strain," International Journal of Solids and Structures, vol. 41, no. 18-19, pp. 5327-5350, 2004.

[22] W. B. Shangguan and Z. H. Lu, "Experimental study and simulation of a hydraulic engine mount with fully coupled fluid-structure interaction finite element analysis model," Computers and Structures, vol. 82, no. 22, pp. 1751-1771, 2004.

[23] W. B. Shangguan and Z. H. Lu, "Modelling of a hydraulic engine mount with fluid-structure interaction finite element analysis," Journal of Sound and Vibration, vol. 275, no. 1-2, pp. 193-221, 2004.

[24] A. Duster, S. Hartmann, and E. Rank, "p-FEM applied to finite isotropic hyperelastic bodies," Journal of Computational Methods In Applied Mechanics and Engineering, vol. 192, no. 47-48, pp. 5147-5166, 2003.

[25] L. A. D. Filho and A. M. Awruch, "Geometrically nonlinear static and dynamic analysis of shells and plates using the eightnode hexahedral element with one-point quadrature," Journal of Finite Elements in Analysis and Design, vol. 40, no. 11, pp. 1297-1315, 2004.

[26] G. Kim and R. Singh, "Nonlinear analysis of automotive hydraulic engine mount," ASME Journal of Dynamic Systems, Measurement and Control, vol. 115, no. 3, pp. 482-487, 1993.

[27] G. Kim and R. Singh, "A study of passive and adaptive hydraulic engine mount systems with emphasis on non-linear characteristics," Journal of Sound and Vibration, vol. 179, no. 3, pp. 427-453, 1995.

[28] A. H. Nayfeh and D. Mook, Nonlinear Oscillations, John Wiley \& Sons, New York, NY, USA, 1993.

[29] A. H. Nayfeh, Introduction to Perturbation Techniques, John Wiley \& Sons, New York, NY, USA, 1993. 

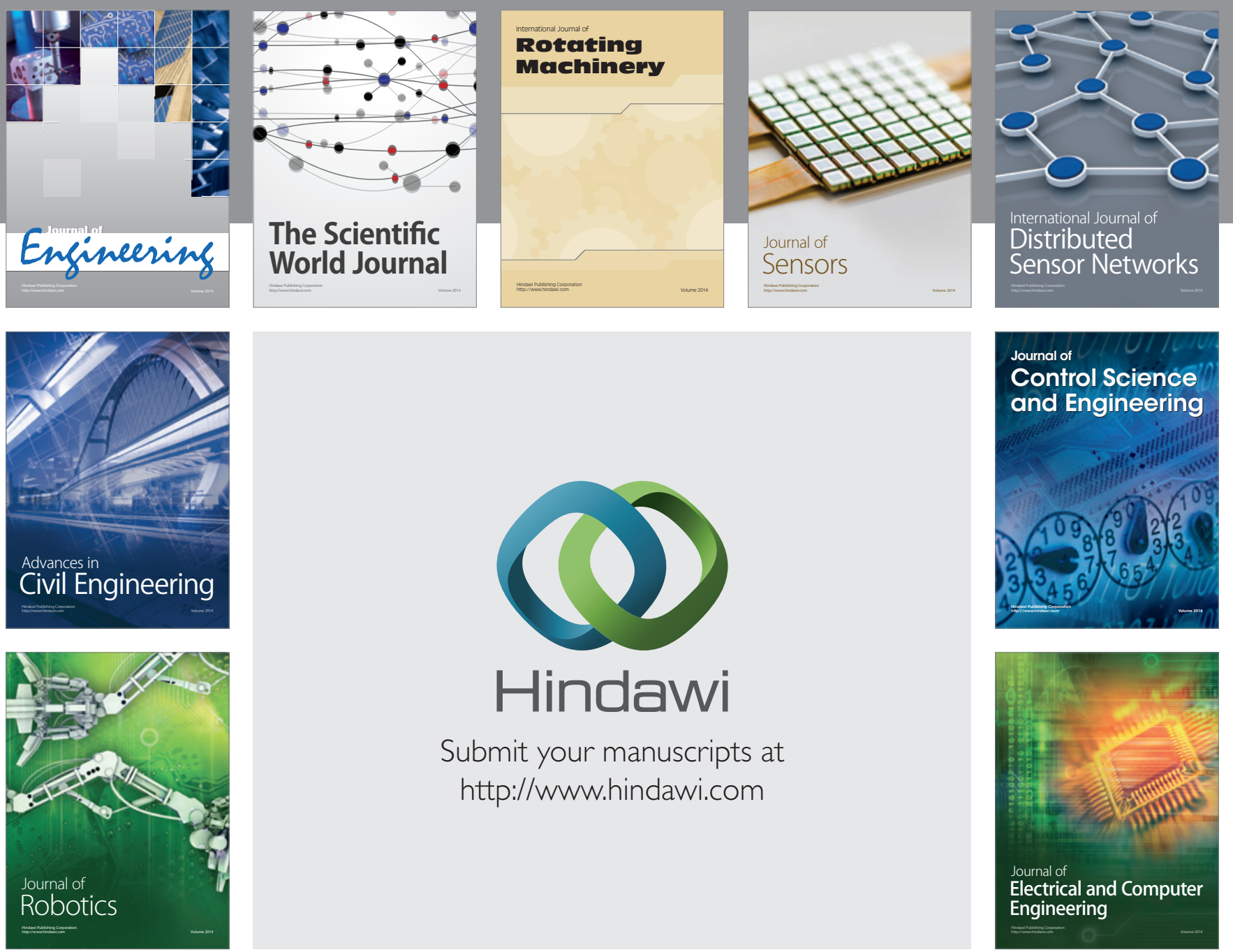

Submit your manuscripts at

http://www.hindawi.com
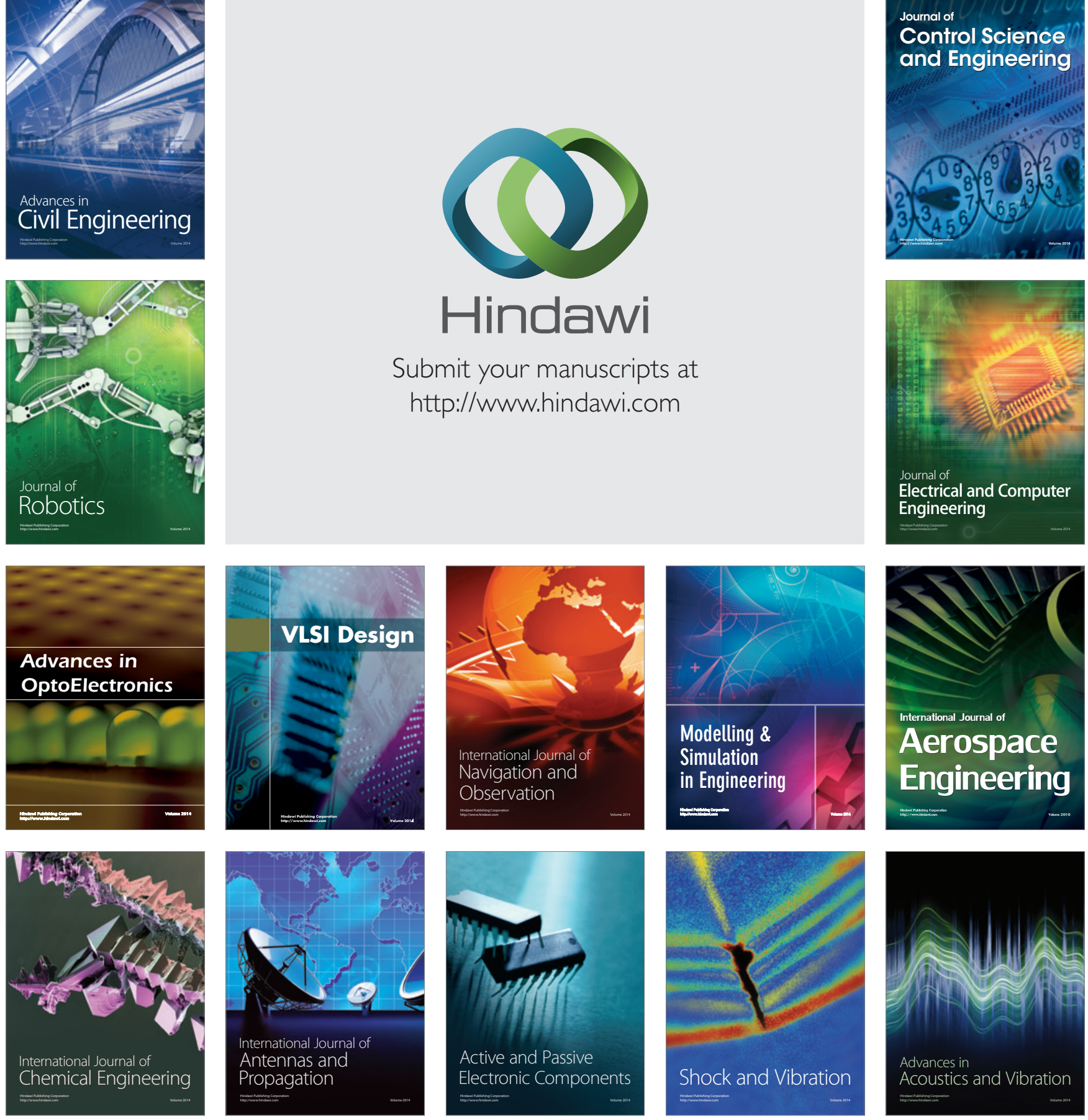